\title{
Electron heat conduction in the solar wind: transition from Spitzer-Härm to the collisionless limit
}

\author{
S. D. Bale ${ }^{1,2}$, M. Pulupa ${ }^{2}$, C. Salem ${ }^{2}$, C. H. K. Chen ${ }^{2}$, and E. Quataert ${ }^{3,1}$
}

\begin{abstract}
We use a statistically significant set of measurements to show that the field-aligned electron heat flux $q_{\|}$in the solar wind at $1 \mathrm{AU}$ is consistent with the Spitzer-Härm collisional heat flux $q_{s h}$ for temperature gradient scales larger than a few mean free paths $L_{T} \gtrsim 3.5 \lambda_{f p}$. This represents about $65 \%$ of the measured data and corresponds primarily to high $\beta$, weakly collisional plasma ('slow solar wind'). In the more collisionless regime $\lambda_{f p} / L_{T} \gtrsim 0.28$, the electron heat flux is limited to $q_{\|} / q_{0} \sim 0.3$, independent of mean free path, where $q_{0}$ is the 'free-streaming' value; the measured $q_{\|}$does not achieve the full $q_{0}$. This constraint $q_{\|} / q_{0} \sim 0.3$ might be attributed to waveparticle interactions, effects of an interplanetary electric potential, or inherent flux limitation. We also show a $\beta_{e}$ dependence to these results that is consistent with a local radial electron temperature profile $T_{e} \sim r^{-\alpha}$ that is a function of the thermal electron beta $\alpha=\alpha\left(\beta_{e}\right)$ and that the $\beta$ dependence of the collisionless regulation constraint is not obviously consistent with a whistler heat flux instability. It may be that the observed saturation of the measured heat flux is a simply a feature of collisional transport. We discuss the results in a broader astrophysical context.
\end{abstract}

Subject headings: Sun: - solar wind, stars: winds, outflows

\section{Introduction}

Thermal conduction in the solar wind provides an important mode of energy transport and determines in part the radial electron temperature profile. The conductive, magnetic field-aligned electron heat flux is defined as $q_{\|}=-\kappa_{\|} \nabla_{\|} T_{e}$ where $\kappa_{\|}$is the thermal conductivity coefficient and $T_{e}(r)$ is the electron temperature. In a fully collisional plasma, the Spitzer-Härm thermal conductivity (Spitzer and Härm 1953) is $\kappa_{\|}=\kappa_{S H} \sim$ $3.16 n_{e} T_{e} \tau_{e} / m_{e}$. Spitzer-Härm (SH) theory assumes that the electron distribution function $f(\vec{v})$ remains approximately Maxwellian as it evolves through a temperature gradient scale, which corresponds to assuming that the Knudsen number $K \sim \lambda_{f p} / L_{T}$ is a small parameter, where $L_{T}=T_{e} /\left|\partial T_{e} / \partial r\right|=R / \alpha$ for $T(r) \sim r^{-\alpha}$, where $\mathrm{R}=1 \mathrm{AU}$, and $\lambda_{f p}=v_{e} \tau_{e}$ is the mean free

\footnotetext{
${ }^{1}$ Physics Department, University of California, Berkeley.

${ }^{2}$ Space Sciences Laboratory, University of California, Berkeley.

${ }^{3}$ Astronomy Department, University of California, Berkeley.
}

path. In particular, SH theory assumes that the distribution function $f \approx f_{0}+f_{1}$, with $f_{0}$ an isotropic Maxwellian and $f_{1}$ an anisotropic $(\cos \theta)$ perturbation, such that $f_{1} \leq f_{0}$ and dropping $\mathcal{O}\left(f_{1}^{2}\right)$ terms (Spitzer and Härm 1953). The electron collision time $\tau_{e}$ goes like $T_{e}^{3 / 2} / n_{e}$, therefore the thermal conductivity scales like $\kappa_{S H} \propto T_{e}^{5 / 2}$. Thus to maintain a constant conductive luminosity $L=4 \pi r^{2} q_{\|}$, the wind must have $T_{e}(r) \sim r^{-2 / 7}$. Measurements of the solar wind electron temperature profile generally show a power law profile $T_{e}(r) \sim r^{-\alpha}$ with values of $\alpha$ from 0.2 to 0.7 (e.g. Marsch et al. 1989), not inconsistent with $\alpha=2 / 7 \approx 0.286$. Of course, energy input from turbulent dissipation is likely to be important as well.

The solar wind electron population at $1 \mathrm{AU}$ consists primarily of a cool Maxwellian 'core' ( $\sim 10$ $\mathrm{eV}, \sim 95 \%$ density), a suprathermal 'halo' ( $\sim 70$ $\mathrm{eV}, \sim 4 \%$ density), and an antisunward 'strahl' population ( $\sim 100-1000 \mathrm{eV}, \sim 1 \%$ density). The core is nearly isotropic, while the halo and strahl 
exhibit clear temperature anisotropies. Since the electrons are subsonic $\left(v_{e} \gg v_{s w}\right)$, the maximum available heat flux corresponds to transport of the full thermal energy $3 / 2 n_{e} k_{b} T_{e}$ at the thermal speed $v_{e}$; this is often called the 'free-streaming' or saturation heat flux $q_{0}=3 / 2 n_{e} k_{\mathrm{B}} T_{e} v_{e}$ (Parker) 1964; Roxburgh 1974; Cowie and McKee 1977). The ratio $q_{s h} / q_{0}$ is proportional to the Knudsen number $K \sim \lambda_{f p} / L_{T}$, the small parameter in SH theory (Cowie and McKee 1977; Salem et al. 2003),

$$
\frac{q_{s h}}{q_{0}}=\frac{-\kappa_{S H} \nabla_{\|} T_{e}}{(3 / 2) n k_{b} T_{e} v_{e}} \approx 1.07 \frac{\lambda_{f p}}{L_{T}}
$$

and this scaling of $q_{\|} / q_{0}$ with $\lambda_{f p} / L_{T}$ provides a clear test of SH theory. Assuming a value of $\alpha$ in $L_{T}$, we can compute the measured $q_{\|} / q_{0}$ vs $\lambda_{f p} / L_{T}$ and compare to Equation (1) for $q_{s h} / q_{0}$; the only free parameter is $\alpha$, the temperature gradient exponent.

In this short paper we show that the measured $q_{\|}$in the solar wind is consistent with $q_{s h}$ until $\lambda_{f p} / L_{T}$ becomes as large at 0.3 . Beyond that, in the collisionless regime, we find that $q_{\|} \sim 0.3 q_{0}$ independent of mean free path. Collisionless regulation of heat flux has been discussed in the context of wave-particle interactions (Gary et al. 1994) and escape from an interplanetary electric potential (Perkins 1973; Hollweg 1974). We divide our data into intervals of electron thermal $\beta_{e}$ (ratio of electron thermal pressure to magnetic field pressure) and compare to theoretical threshold values for whistler and magnetosonic instability constraints on heat flux (Gary et al. 1994). We find that the whistler instability overconstrains the measurements while the magnetosonic instability is more consistent. We also find that the data fit better to the $\mathrm{SH}$ relationship (in the collisional regime) if there is a $\beta_{e}$ dependence to the temperature profile index $\alpha$. This may reflect the $\beta_{e}$ dependence of a wave heating mechanism, or may be a proxy for another parameter, such as collisional age.

\section{Measurements}

We use measurements of the solar wind electron distribution function from the Three Dimensional Plasma (3DP) instrument (Lin et al. 1995; Pulupa et al. 2013) on the NASA Wind space- craft. The 3DP instrument uses two separate sensors - EESA-L and EESA-H - to measure the full 3D distribution function $f(\vec{v})$ in 88 angular bins from $\sim 1 \mathrm{eV}$ to $\sim 30 \mathrm{keV}$, once per spacecraft spin $(3 \mathrm{~s})$. Each EESA sensor is a 'top hat' electrostatic analyzer (Carlson et al. 1982) designed to measure solar wind thermal electrons (EESA-L) and suprathermals (EESA-H) each in 15 log-spaced energy steps (few eV to $1.1 \mathrm{keV}$ for EESA-L and 100 $\mathrm{eV}$ to $30 \mathrm{keV}$ for EESA-H). Measurements from the two detectors are combined to form a single distribution function and this distribution function is corrected for spacecraft floating potential effects using quasi-thermal noise measurements as an absolute plasma density benchmark; low energy monopole corrections (few Volts) are less important for higher-order moments such as heat flux, however dipole fields may introduce errors of $5 \%$ to the heat flux moment (Pulupa et al. 2013). We use 155,182 independent measurements of $f(\vec{v})$ from two, 2-year intervals: 1995-1997 (solar minimum) and 2001-2002 (solar maximum) and include only 'ambient' solar wind (no CMES, foreshock, etc.). Intervals of 'bi-directional' heat flux (usually associated with CMEs) are also excluded. We compute the electron heat flux moment as

$$
\vec{q}_{e}=\frac{1}{2} \int d v^{3} f(\vec{v}) w^{2} \vec{w}
$$

from the measurements, where the secular velocity is $\vec{w}=\vec{v}-\vec{v}_{b}$ and $\vec{v}_{b}$ is the bulk speed. Here we consider the (dominant) magnetic field-aligned component of the heat flux $q_{\|}=\vec{q} \cdot \hat{B}$. The thermal electron properties $q_{0}, \lambda_{f p}$, and $\beta_{e}$ are computed using measurements of the 'core' electron population, a point that we discuss below.

Figure 1 shows the joint probability distribution of $q_{\|} / q_{0}$ and $\lambda_{f p} / L_{T}$ normalized to the peak value in each $\lambda_{f p} / L_{T}$ histogram and Equation (1) is over plotted as a diagonal line in the top panel. The number of points in each $\lambda_{f p} / L_{T}$ bin is shown in the lower panel and a temperature exponent of $\alpha \sim 2 / 7$ is used to calculate $L_{T}$. It is apparent that $q_{\|} / q_{0}$ tracks Equation (1) over much of its range.

Figure 2 shows the mode (most probable value) in each bin of $\lambda_{f p} / L_{T}$. The modes are calculated directly from the distribution of data (red points) and from log-normal fits (black points). In this Figure and in Figure 1, the Spitzer-Härm rela- 


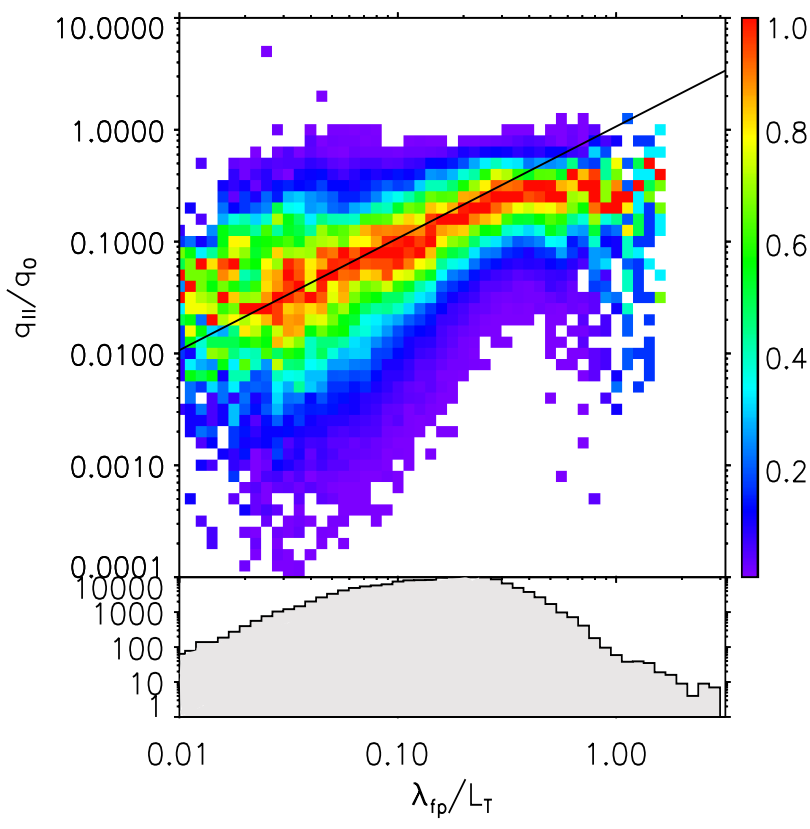

Fig. 1.- Joint normalized distribution of the normalized electron heat flux $q_{\|} / q_{0}$ and the temperature Knudsen number $K=\lambda_{f p} / L_{T}$ (top panel) and the distribution of data with $\lambda_{f p} / L_{T}$ (bottom panel). The diagonal line in the top panel is the Spitzer-Härm relationship (Equation (1)). tionship in Equation (1) appears to be a reasonably good approximation to the data. At about $\lambda_{f p} \simeq 0.28 L_{T}$, the measured data breaks from the SH line and flattens to a fixed value of $q_{\|} \sim 0.29 q_{0}$. Approximately $65 \%$ of our measurements lie in the SH regime, the other $35 \%$ in the collisionless regime $q_{\|} \simeq 0.29 q_{0}$ (bottom panel of Figure 2). The so-called 'collisional age' $A=v_{s w} \tau$ is often used to measure collisional evolution (Salem et al. 2003; Bale et al. 2009), especially when considering interactions with ions convecting at the solar wind speed $v_{s w}$. The collisional age correlates strongly with solar wind speed; fast solar wind is more collisionless (hot and rarified) and slow wind is more collisional (cooler and dense); therefore data in Figure 1 and 2 in the collisional regime $\left(\lambda_{f p} / L_{T} \ll 1\right)$ is primarily slow wind, while collisionless data $\left(\lambda_{f p} / L_{T} \gg 1\right)$ is primarily fast wind.

\section{Electron $\beta$ dependence}

In Figure 3, we break this data into 4 intervals of electron thermal beta $\beta_{e}=\left(n_{e} k_{\mathrm{B}} T_{e}\right) /\left(B^{2} / 2 \mu_{0}\right)$. Since $\lambda_{f p} / L_{T} \propto 1 / n_{e}$ and density variations dominate the pressure variations, the high $\beta_{e}$ plasma corresponds to collisional plasma (small $\lambda_{f p} / L_{T}$ ). This evolution can be seen in the panels of Figure 3 , organized from high to low $\beta_{e}$ (top to bottom); the points move towards the right (towards the collisionless regime). As the clusters of points move to the right, they maintain the SH-like power law behavior, but require different values of $\alpha$ (the temperature profile index) to conform to the curve - note that $\alpha$ is the only free parameter. Alignment to the $\mathrm{SH}$ curve give corrected values and suggest a $\beta_{e}$ dependence to the electron temperature profile index $\alpha$ and to the breakpoint $\Lambda_{b}$ between the collisional $\mathrm{SH}$ and the collisionless heat flux regimes. Table 1 summarizes these results.

Figure 3 also shows the $\beta_{e}$-dependent instabilitylimited electron heat flux values for both the magnetosonic and whistler instabilities as calculated by Gary et al. (1994). We use most probable values of $\beta_{e}$ in each interval and scale the corresponding $\left(\gamma_{\max }=10^{-3} \Omega_{p}\right)$ threshold from Figure 1 of Gary et al. (1994): magnetosonic-limited values are shown as horizontal dotted red lines, while whistler instability-limited heat flux is shown as blue dotted lines. It can be seen that the heat fluxdriven whistler instability overconstrains the data; 


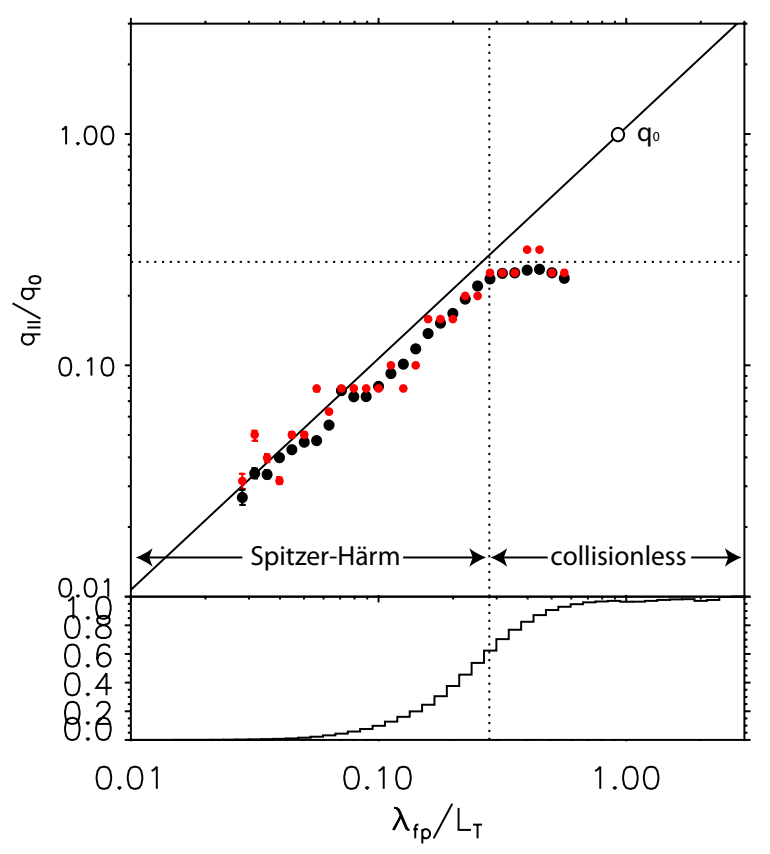

Fig. 2.- Plot of the most probable values (modes) of $q_{\|} / q_{0}$ in bins of $\lambda_{f p} / L_{T}$ with standard error errorbars (very small). Red points are the computed modes, black points are modes determined from $\log$ normal fits. Again, the black diagonal line in the upper panel is the SH Equation (1). The measured values depart from $\mathrm{SH}$ behavior above $\lambda_{f p} / L_{T} \sim q_{\|} / q_{0} \gtrsim 0.28$. The cumulative distribution in the bottom panel shows that approximately $65 \%$ of this dataset corresponds to SH heat flux. A symbol at $q_{\|}=q_{0}$ show the free-streaming value.

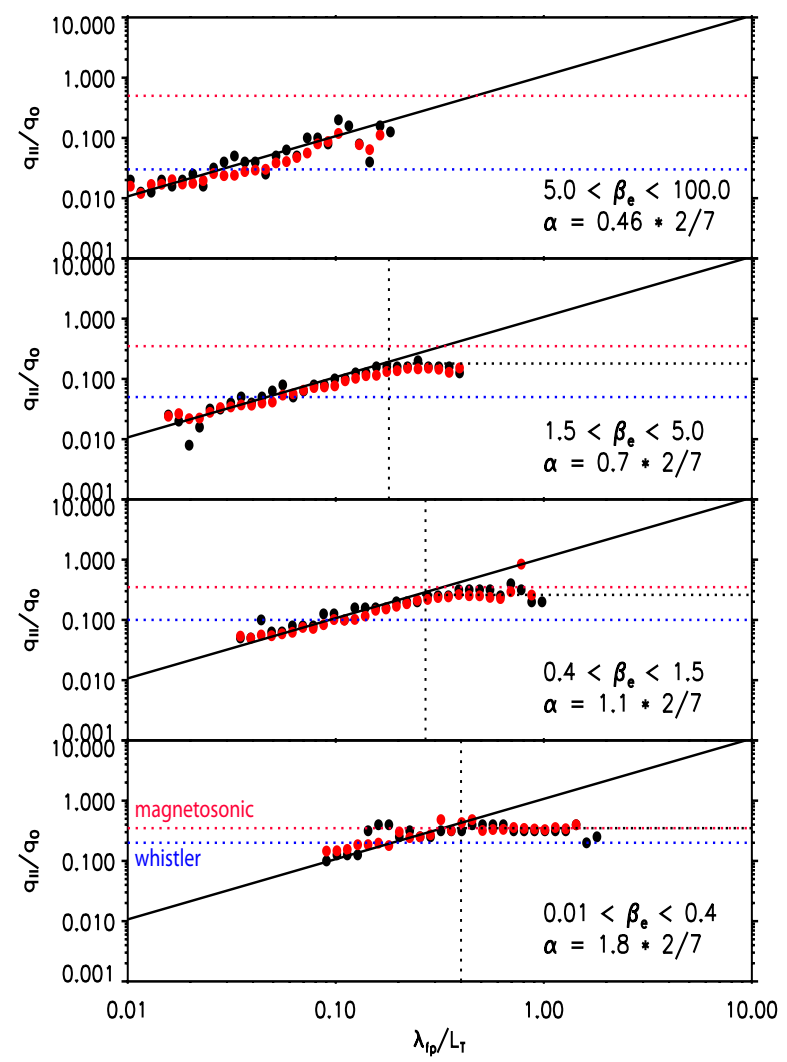

Fig. 3.- Data from Figure 2 broken into four intervals of electron thermal $\beta_{e}$. In each interval, the data are consistent with the $\mathrm{SH}$ relationship with the temperature gradient index $\alpha$ as a free parameter. The $\beta_{e}$ dependence of $\alpha$ is summarized in Table 1. Dotted horizontal lines are the thresholds of the magnetosonic instability (red) and whistler instability (blue), both from Gary et al (1994). The whistler instability appears inconsistent with the heat flux levels in the collisionless regime. 
the magnetosonic instability underconstrains the data in the $\mathrm{SH}$ regime, but is in fact consistent with the limiting electron heat flux in the collisionless regime.

\begin{tabular}{|c|c|c|c|}
\hline$\beta_{e}$ & $\mathrm{~N}$ & $\alpha$ & $\Lambda_{b}$ \\
\hline $0.01-0.4$ & 12,507 & 0.51 & 0.40 \\
$0.4-1.5$ & 50,056 & 0.31 & 0.27 \\
$1.5-5.0$ & 80,560 & 0.20 & 0.18 \\
$5-100$ & 12,059 & 0.13 & - \\
\hline
\end{tabular}

Table 1: The $\beta_{e}$ dependence of $T_{e}(r) \sim r^{-\alpha}$ and the SH-collisionless transition scale $\Lambda_{b} L_{T} . \mathrm{N}$ is the number of measurements in each $\beta_{e}$ interval. Note that the limiting heat flux in the collisionless regime is proportional to the breakpoint $q_{\|} \simeq 1.07 \Lambda_{b} q_{0}$. Also note that $2 / 7 \approx 0.286$.

\section{Conclusions}

In this manuscript, we show that the transition from collisional (Spitzer-Härm) to collisionless electron heat conduction in the solar wind occurs at a mean free path of about $\lambda_{f p} \simeq 0.28 L_{T}$, where $L_{T}$ is the electron temperature gradient scale. In the collisionless regime, the heat flux is limited to $q_{\|} \sim 0.3 q_{0}$, where $q_{0}$ is the free-streaming value. Some previous analyses suggested departures from the SH value (Feldman et al. 1975; Pilipp et al. 1987). This could be attributed to the choice of data intervals in those analyses, none of which were very statistical. In fact, a preliminary statistical analysis of the Helios electro heat flux measurements shows similar results to what we present here (K. Horaites, private communication).

In our analysis, we have used the 'core' electron density and temperature to compute $q_{0}$ and $\lambda_{f p}$. Theoretical work on heat conduction on steep temperature gradients has shown that a self-consistent flux limitation arises as SH theory begins to break down (for $f_{1} \sim f_{0}$ ) and that this corresponds to order $\lambda_{f p} / L_{T} \sim 0.1$ (Shvarts et al. 1981), similar to our results. The growing departure of the measurements from the SH curve in our Figure 2 is consistent with this effect (which can be seen by multiplying Figure 2 of (Shvarts et al. 1981) by $\left.\lambda_{f p} / L_{T}\right)$. Since $f \sim n / T^{3 / 2}$, the constraint $f_{1} \leq f_{0}$ is well-satisfied for solar wind parameters $\left(n_{h} \sim 1 / 20 n_{c}\right.$ and $\left.T_{h} \sim 7-10 T_{c}\right)$ for ve- locities less than about $v \lesssim 2.6 v_{t h, c}$, therefore the core population does represent the collisional physics. Similar results were obtained by Smith et al. (2012), who solved the electron kinetic equation with a linearized Fokker-Planck operator in a fixed ion profile and found limiting heat flux values comparable to ours and attribute it to skewness in the distribution function at speeds of $v \geq 3 v_{t h}$. A simple model of a suprathermal tail escaping from an interplanetary electric field (Hollweg 1974) predicts a collisionless heatflux $q \sim 3 / 2 n_{e} k_{\mathrm{B}} T_{e} v_{s w}$, which is also approximately consistent with our results, since $v_{s w} \sim 1 / 3 v_{e}$. If we repeat our analysis with the full electron temperature $T_{\text {all }}=$ $\left(n_{c} T_{c}+n_{h} T_{h}+n_{s} T_{s}\right) /\left(n_{c}+n_{h}+n_{s}\right)$, we find a similar breakpoint, but somewhat faster departure from SH.

Our measurements show that the solar wind heat flux is well-described by the collisional Spitzer-Härm value until the mean free path is approximately one third of the temperature gradient scale $\lambda_{f p} \sim 1 / 3 L_{T}$ and for larger $\lambda_{f p}$ is proportional to the saturation value $q \sim 1 / 3 q_{0}$. While the limiting mechanism is not yet understood, these results should be useful for solar wind and coronal modeling efforts. Our analysis also suggests that the $\beta_{e}$-dependence of whistler heat flux instability (Gary et al. 1994) is inconsistent with the data, however a magnetosonic instability may be consistent in the collisionless regime. We also infer a $\beta_{e}$-dependance to the temperature profile index $\alpha$, which may indicate an additional energy transport process, or be a proxy for another plasma parameter (e.g. Mach number, collisionality, etc.).

The observation that the electron heat flux remains 'classical' to Knudsen numbers as large as $K_{T} \sim 0.3$ may have implications for solar wind models which transition from fluid to exospheric domains (viz. Echim et al. 2011 and references therein) and for overall heat transport in the corona. If the electron temperature gradient is only a weak function of radial distance (in the free solar wind), then $\lambda_{f p}$ will be smaller in the inner heliosphere, and it may be that most of the solar wind lies in the $\mathrm{SH}$ regime there.

Electron thermal conduction, in both the collisional Spitzer-Härm and collisionless limits, is energetically and dynamically important in other low-collisionality astrophysical plasmas, including, 
e.g., the hot intracluster medium in galaxy clusters (e.g. Bertschinger and Meiksin 1986), the hot interstellar medium in galaxies (Cowie \& McKee 1977), and some accretion disks around neutron stars and black holes (e.g. Sharma et al. 2006). These astrophysical plasmas are probably characterized by $\beta_{e} \sim 1-10$, not too dissimilar from a large fraction of the epochs of in situ solar wind data used here (Table 1). Our results suggest that parallel thermal conduction is likely to be comparable to the Spitzer-Härm and/or saturated values in these systems. In more detail, the results in Fig. 3 and Table 1 could be used for modeling parallel thermal conduction in other astrophysical contexts. Of course, the global magnetic field geometry determines in part how the parallel thermal conductivity translates into large-scale redistribution of heat (e.g., Chandran \& Maron 2004), and the field geometry is significantly more uncertain in these other astrophysical contexts.

This work was supported in part by NSF grant AGS 0962726 (SHINE) and NASA HTP grant NNX11AJ37G.

\section{REFERENCES}

Bale, S. D., J. C. Kasper, G. G. Howes et al., Phys. Rev. Lett., 103, 211101, 2009.

Bertschinger, E. and A. Meiksin, ApJ, 306, L1, 1986.

Carlson, C. W., D. W. Curtis, G. Paschmann et al., Adv Space Res., 2, 67, 1982.

Chandran, B. and J. Maron, ApJ, 602, 170, 2004.

Cowie, L. L., and C. F. McKee, ApJ, 2111, 135, 1977.

Echim, M. M., J. Lemaire, and O. Lie-Svendsen, Surv. Geophys., 32:1-70, 2011.

Feldman, W. C., J. R. Ashbridge, S. J. Bame, et al., J. Geophys. Res., 80, 4181, 1975.

Lin, R. P., K. A. Andeerson, S. Ashford et al., Space Sciences Reviews, 71, 125, 1995.

Gary, S. P., E. E. Scime, J. L. Phillips, et al., JGR, 99, 23391,1994.

Hollweg, J. V., J. Geophys. Res., 79, 3845, 1974
Parker, E. N., ApJ, 139, 93, 1964.

Perkins, F., ApJ, 179, 637, 1973.

Marsch E., K. M. Thieme, H. Rosenbauer et al., J. Geophys. Res., 94, 6893, 1989.

Pilipp, W. G., K.-H. Muehlhauser, H. Miggenrieder, et al, J. Geophys. Res., 92, 1075, 1987

Pulupa, M., S. D. Bale, C. Salem et al., submitted to J. Geophys. Res., 2013.

Roxburgh, I. W., Solar Phys., 35, 481, 1974.

Salem, C., D. Hubert, C. Lacombe et al., ApJ, 585, 1147, 2003.

Shvarts, D., J. Deletttrez, R. McCrory, et al., Phys. Rev. Lett., 47, 247, 1981.

Sharma, P., G. Hammett, E. Quataert, et al., ApJ, 637, 952, 2006.

Smith, H. M., E. Marsch, and P. Helander, ApJ, 753:31, 2012.

Spitzer L. and R. Härm, Phys Rev, 89, 5, 977, 1953.

This 2-column preprint was prepared with the AAS LATEX macros v5.2. 